\title{
ARNOLD CONJECTURE FOR CLIFFORD SYMPLECTIC PENCILS
}

\author{
VIKTOR L. GINZBURG AND DORIS HEIN
}

\begin{abstract}
We establish a version of the Arnold conjecture, both the degenerate and non-degenerate cases, for target manifolds equipped with Clifford pencils of symplectic structures and the domains (time-manifolds) equipped with frames of divergence-free vector fields meeting a certain additional requirement. This result generalizes the original work on the hyperkähler Arnold conjecture by Hohloch, Noetzel and Salamon for three-dimensional time and also the previous work by the authors.
\end{abstract}

\section{Contents}

1. Introduction 1

Acknowledgments 2

2. Arnold Conjecture for Symplectic Pencils 2

2.1. Symplectic Pencils 2

2.2. Action Functional 4

2.3. Arnold Conjecture 5

3. Regularity and the Clifford Condition 6

3.1. Examples of Regular Frames 6

3.2. Clifford Condition Revisited 8

4. Proof of Theorem 2.2 9

References 13

\section{INTRODUCTION}

We prove an analog of the Arnold conjecture in the setting where the onedimensional time, $S^{1}$ or $\mathbb{R}$, is replaced by a multi-dimensional time manifold $M$. To be more specific, the time manifold $M$ is now any closed manifold equipped with a frame of diversion-free vector fields satisfying a certain regularity requirement. The target manifold $W$ (the space) is equipped with a Clifford pencil (i.e., a linear space with some additional properties) of symplectic structures. The space of nullhomotopic maps from $M$ to $W$ carries a suitably defined action functional and, in the spirit of the classical Arnold conjecture, the number of its critical points is bounded from below by a certain topological invariant of $W$. As in the Hamiltonian Arnold conjecture (or in Morse and Ljusternik-Schnirelman theories), this is the

Date: October 31, 2018.

2000 Mathematics Subject Classification. 53D40, 32Q15.

The work is partially supported by the NSF and by the faculty research funds of the University of California, Santa Cruz. 
sum of Betti numbers in the non-degenerate case and the cup-length plus one for general Hamiltonians.

Our result generalizes and builds on the results of Hohloch, Noetzel and Salamon, [HNS], and the previous results of the authors, [GH]. In [HNS], a similar version of the Arnold conjecture is established in the non-degenerate case for a flat hyperkähler target manifold and the time manifold $\mathbb{T}^{3}$ or $\mathrm{SU}(2)$ by means of Floer theory. In [GH], the degenerate case is treated as well and, furthermore, for $M=\mathbb{T}^{r}$, the proof of the Arnold conjecture results is extended to all flat target manifolds carrying a Clifford pencil of symplectic structures. Hence, the main new point of the present paper is a further extension of the argument. We now consider any closed time manifold equipped with a regular divergence-free frame, replacing the Lie group structures of $\mathrm{SU}(2)$ and $\mathbb{T}^{r}$ with translation-invariant frames. The regularity requirement is simply the condition that the critical points of the action functional for the zero Hamiltonian are exactly the constant maps.

Our proof differs crucially from the Floer theoretic argument in [HNS] and technically from the argument in $[\mathrm{GH}]$. As in [GH], it relies on a finite-dimensional approximation method combined with Morse or Ljusternik-Schnirelman theory for generating functions along the lines of [CZ]. However, in contrast with previous works using a similar technique with Lie groups serving as time manifolds (as, e.g., in $[\mathrm{CZ}, \mathrm{GH}])$, we do not explicitly determine the matrix representations for the (unperturbed) $L^{2}$-gradient $\phi$ of the action functional by means of the Fourier expansion on $S^{1}$ or its counterpart for Lie groups provided by the Peter-Weyl theorem. Instead, we use the ellipticity of $\not$ to decompose the function space into the sum of finite-dimensional eigenspaces, which turns out to be sufficient for the finite-dimensional reduction method to apply.

Acknowledgments. The authors are deeply grateful to Dietmar Salamon for calling their attention to the regularity problem. They also would like to thank Jie Qing, Claude Viterbo, and Siye Wu for useful discussions. A part of this work was carried out while the first author was visiting the Institute for Advanced Study during the Symplectic Dynamics program and he would like to thank the Institute for its warm hospitality and support.

\section{Arnold Conjecture for Symplectic Pencils}

2.1. Symplectic Pencils. A symplectic pencil on a finite-dimensional, real vector space $V$ is a linear subspace $\mathcal{S} \subset \bigwedge^{2} V^{*}$, each element of which, except of course 0 , is a symplectic structure on $V$. Alternatively, we say that skew-symmetric bilinear forms $\omega_{1}, \ldots, \omega_{r}$ on $V$ generate a symplectic pencil $\mathcal{S}$ when all non-zero linear combinations $\sum_{l} \lambda_{l} \omega_{l}$, forming $\mathcal{S} \backslash 0$, are symplectic forms. In what follows, we call $\operatorname{dim} \mathcal{S}$ the rank of the pencil and assume that $\left\{\omega_{1}, \ldots, \omega_{r}\right\}$ is a basis of $\mathcal{S}$.

Consider, for instance, the Clifford algebra $C l_{r}$ of a positive-definite quadratic form on $\mathbb{R}^{r}$ (We refer the reader to [LM] or, e.g., [HP, Chap. 2], for a discussion of Clifford algebras; note that here, in contrast with [GH], we use the conventions of [LM].) Let $V$ be a real $C l_{r}$-module, i.e., an (orthogonal) representation of $C l_{r}$. This is simply a collection of $r$ complex structures $J_{1}, \ldots, J_{r}$ on $V$ (corresponding to an orthonormal basis in $\mathbb{R}^{r}$ ), which anti-commute and are all compatible with the same inner product $\langle$,$\rangle . In other words, the operators J_{l}$ are $\langle$,$\rangle -orthogonal,$ 
$J_{l}^{2}=-I$ for all $l$, and

$$
J_{l} J_{j}+J_{j} J_{l}=0 \text { whenever } l \neq j .
$$

Then the forms

$$
\omega_{l}(X, Y):=\left\langle J_{l} X, Y\right\rangle
$$

generate a symplectic pencil $\mathcal{S}$ of rank $r$ on $V$. To see this, note that $\omega=\sum \lambda_{l} \omega_{l}$ is symplectic if and only if $J=\sum_{l} \lambda_{l} J_{l}$ is non-degenerate. This is the case when not all $\lambda_{l}=0$, since $J^{2}=-\left(\sum_{l} \lambda_{l}^{2}\right) I$ due to (2.1). In particular, $\omega \neq 0$, and hence $\left\{\omega_{l}\right\}$ is a basis of $\mathcal{S}$. Thus, the rank of $\mathcal{S}$ is indeed $r$. We will refer to these pencils (equipped in addition with the basis $\omega_{l}$ ) as Clifford symplectic pencils.

Among Clifford symplectic pencils are, for example, the symplectic pencils associated with hyperkähler structures. In this case, $r=3$, and the complex structures $J_{l}$ are $\langle$,$\rangle -orthogonal and satisfy the quaternionic relations, i.e., in addition to (2.1),$ we also have $J_{1} J_{2}=J_{3}$.

Clearly, when $V$ admits a symplectic pencil $\mathcal{S}$ of rank $r$, it also admits pencils of rank smaller than $r$, e.g., subpencils of $\mathcal{S}$. Conversely, in some instances, a given pencil can be extended to a pencil of higher rank. For example, a Clifford pencil of rank two extends to a hyperkähler pencil by setting $J_{3}=J_{1} J_{2}$. As a consequence, whenever $V$ carries a Clifford pencil $\mathcal{S}$ of rank three, it also carries a hyperkähler pencil, in general different from $\mathcal{S}$, obtained by, e.g., keeping $J_{1}$ and $J_{2}$ intact and replacing $J_{3}$ with $J_{1} J_{2}$. Finally note that, as is not hard to see, symplectic pencils of rank $r$ form an open subset (possibly empty) in the Grassmannian of $r$-dimensional linear subspaces in $\bigwedge^{2} V^{*}$.

A vector space $V$ admits a symplectic pencil of rank $r$ if and only if the unit sphere in $V$ admits $r$ point-wise linearly independent vector fields. To see this, let us view a symplectic pencil $\mathcal{S}$ as a pencil of non-degenerate Poisson structures on the dual space $V^{*}$. Fix a positive definite quadratic form $K: V^{*} \rightarrow R$. Let $X_{l}$ be the Hamiltonian vector field of $K$ on the sphere $\Sigma=\{K=1\} \subset V^{*}$ with respect to the Poisson structure $\omega_{l}$. Then the non-degeneracy of $\sum_{l} \lambda_{l} \omega_{l}$ readily implies that the vector fields $X_{l}$ are point-wise linearly independent on $\Sigma$. This shows that the rank of $\mathcal{S}$ is no greater than the number of linearly independent vector fields on $\Sigma$. The opposite inequality comes from Clifford pencils, cf. [Hu, Chap. 12]. Finally, recall how the maximal value $r$ of linearly independent vector fields on the sphere in $V$ is determined by the dimension of $V$. Let $\operatorname{dim} V=2^{4 d+c} b$, where $d \geqslant 0$ and $0 \leqslant c \leqslant 3$ are integers and $b$ is odd. Then $r=8 d+2^{c}-1$; see, e.g., [Hu, Chap. 12 and 16] and, in particular, pp. 152-154 therein.

Remark 2.1. One can construct examples of symplectic pencils which are not isomorphic, in the obvious sense, to Clifford pencils. Moreover, a dimension count suggests, although we do not have a complete proof of this fact, that when $r \geqslant 3$, the collection of non-Clifford pencils contains a set which is open and dense in the space of all symplectic pencils. (When $r=2$, the situation appears to be more complicated. It is not inconceivable that Clifford pencils of rank two are structurally stable, i.e., every pencil close to such a Clifford pencil is also Clifford.) Pencils of linear symplectic structures also arise in the study of manifolds equipped with fat fiber bundles introduced in [We1] or of fat distributions; see [Mo, Section 5.6] and references therein, and also [FZ]. Pencils of complex structures are considered in, e.g., [MS, Jo].

We will revisit the Clifford condition in Section 3.2. 
2.2. Action Functional. In this section, we will first define the action functional under the conditions needed for the proof of our main theorem, the Arnold conjecture, and then discuss the role of these conditions in Section 2.2.2.

2.2.1. Definition of the Action Functional. Throughout this subsection, we will assume that $\omega_{1}, \ldots, \omega_{r}$ is a basis of a Clifford symplectic pencil $\mathcal{S}$ on $V$ as above.

Let $W$ be a smooth compact quotient of $V$ by a group of transformations preserving all $\omega \in \mathcal{S}$ and the product $\langle$,$\rangle . (As a consequence, the complex structures J_{l}$ are also preserved.) For instance, $W$ can be the quotient of $V$ by a lattice. (There are, however, other examples; see, e.g., [HNS, p. 2548].)

Furthermore, let us fix a closed manifold $M$ equipped with a volume form $\mu$ and a frame $v=\left\{v_{1}, \ldots, v_{r}\right\}$ of divergence-free vector fields, i.e., $r$ divergence-free vector fields $v_{i}, i=1, \ldots, r$, forming a basis of the tangent space at every point of $M$. The manifold $M$ takes the role of time in Hamiltonian dynamics.

By analogy with Hamiltonian dynamics, a Hamiltonian is a time-dependent function on the target space, i.e., a smooth function

$$
H: M \times W \rightarrow \mathbb{R} .
$$

The action functional $\mathcal{A}_{H}$ is real-valued on the space $\mathcal{E}$ of $C^{\infty}$-smooth (or just $C^{2}$ ), null-homotopic maps $f: M \rightarrow W$. As in [GH], we introduce $\mathcal{A}_{H}$ in two steps. First, let $F:[0,1] \times M \rightarrow W$ be a homotopy between $f$ and the constant map. This is an analog of a capping in the definition of the standard Hamiltonian action functional. The unperturbed action functional is

$$
\mathcal{A}(f)=-\sum_{l} \int_{[0,1] \times M} F^{*} \omega_{l} \wedge i_{v_{l}} \mu .
$$

It is routine to check that $\mathcal{A}(f)$ is well-defined, i.e., independent of $F$. (Here, it would be sufficient to assume that, e.g., the universal covering of $W$ is contractible.) Finally, the total or perturbed action functional is

$$
\mathcal{A}_{H}(f)=\mathcal{A}(f)-\int_{M} H(f) \mu .
$$

For instance, when $r=1$ and $M=\mathbb{T}^{1}$, we obtain the ordinary action functional of Hamiltonian dynamics. In the case of a hyperkähler quotient $W$ and $M=\mathbb{T}^{3}$ or $\mathrm{SU}(2)$, this action functional coincides, up to a sign, with the ones defined in [HNS].

The differential of $\mathcal{A}$ at $f \in \mathcal{E}$ is

$$
(d \mathcal{A})_{f}(w)=\sum_{l} \int_{M} \omega_{l}\left(L_{v_{l}} f, w\right) \mu
$$

where $w \in T_{f} \mathcal{E}$ is a vector field along $f$. Thus, the gradient of $\mathcal{A}$ with respect to the natural $L^{2}$-metric on $\mathcal{E}$ is a Dirac type operator

$$
\nabla_{L^{2}} \mathcal{A}(f)=\sum_{l} J_{l} L_{v_{l}} f=: \not \partial f .
$$

Note that, under our assumptions on $v_{l}$ and $\omega_{l}$, this operator is elliptic and selfadjoint; see Proposition 4.1. This fact is absolutely crucial for the proof of the main theorem of the paper (Theorem 2.2). In what follows, when the dependence of $\phi$ on the frame $v=\left\{v_{1}, \ldots, v_{r}\right\}$ is essential, we will use the notation $\phi_{v}$.

As in [Sa], let us call the operator $\phi$ (or the frame $v$ when $V$ and the pencil are fixed) regular when the only solutions $f: M \rightarrow V$ of the equation $\not f=0$ are 
constant functions. In other words, $W$ is exactly the set of critical points of $\mathcal{A}$. This requirement, playing an important role in the main result of the paper, is further discussed in Section 3.1.

Regardless of the regularity condition, we have

$$
\nabla_{L^{2}} \mathcal{A}_{H}(f)=\not f-\nabla H(f)
$$

where $\nabla H$ denotes the gradient of $H$ along $W$. As a consequence, the critical points of $\mathcal{A}_{H}$ are the solutions $f \in \mathcal{E}$ of the equation

$$
\not f=\nabla H(f) .
$$

At a critical point $f$ of $\mathcal{A}_{H}$, the Hessian $d_{f}^{2} \mathcal{A}_{H}$ is defined in the standard way as the second variation of $\mathcal{A}_{H}$. This is a quadratic form on $T_{f} \mathcal{E}$ equal to the $L^{2}$-pairing with the linearization of $\nabla_{L^{2}} \mathcal{A}_{H}$ at $f$. We call $f$ a non-degenerate critical point when this operator $T_{f} \mathcal{E} \rightarrow T_{f} \mathcal{E}$ is one-to-one, cf. [HNS, p. 2559]. A Hamiltonian $H$ is said to be non-degenerate when all critical points of $\mathcal{A}_{H}$ are non-degenerate. In our setting, non-degeneracy is a generic condition on $H$, i.e., the set of non-degenerate Hamiltonians is residual in $C^{\infty}(M \times W)$. (The proof in [HNS, p. 2574-2576] carries over to our setting with straightforward modifications.)

2.2.2. Requirements. Clearly, the action functional $\mathcal{A}_{H}$ is defined for any manifold $W$ equipped with $r$ symplectic (or even closed) 2-forms and a closed manifold $M$ equipped with a volume form $\mu$ and $r$ divergence-free vector fields. (Of course, as in the case of the classical Hamiltonian action functional, $\mathcal{A}_{H}$ can be multivalued unless $W$ meets some additional requirements or, in other words, its value on $f$ may depend on the choice of capping $F$.) However, in such a general setting, the action functional is probably of little interest. From our perspective, one meaningful condition to impose on $\mathcal{A}_{H}$ or, equivalently, $\mathcal{A}$ is that the operator $\phi$ should be elliptic. To analyze what is essential for this requirement to hold, we focus on the manifolds $W$ and $M$ separately.

Assume first that the vector fields $v_{i}$ form a basis at every point of $M$. Then the operator $\phi$ is elliptic if and only if the forms $\omega_{l}$ generate a symplectic pencil in the space of two-forms $\Omega^{2}(M)$. (However, the forms need not be linearly independent.) For instance, a manifold with a flat symplectic pencil, i.e., the quotient $W$ of a vector space $V$ equipped with a symplectic pencil, meets this requirement. Note that closed manifolds admitting (Clifford) symplectic pencils in $\Omega^{2}(W)$ are extremely rare, cf. [GHJ, Chap. 21]. When $r \geqslant 2$, every such "Clifford" manifold is automatically hyperkähler with the third complex structure being $J_{1} J_{2}$. The authors are not aware of any non-flat example where $r>3$. Yet even flat examples would be of interest in the context of the Arnold conjecture. In fact, so far all available methods for $r \geqslant 2$ require $W$ to be flat; see [GH, HNS].

Looking at the other side of the story, let us assume that the forms $\omega_{l}$ form a basis of a symplectic pencil. It is easy to see that $\not$ cannot be elliptic unless the vector fields $v_{l}$ generate the tangent space to $M$ at every point of $M$, cf. the proof of Proposition 4.1. However, the operator $\phi$ can still be fairly close to elliptic, e.g., hypoelliptic, when the vector fields are bracket-generating.

2.3. Arnold Conjecture. Let now $W$ and $M$ be as in Section 2.2.1. Namely, $W$ is the quotient of a vector space $V$ equipped with a Clifford pencil of rank $r$ by a group of transformations preserving the pencil and the inner product $\langle$,$\rangle ; and let$ 
$M$ be a closed $r$-manifold with a volume form $\mu$ and a frame $v=\left\{v_{1}, \ldots, v_{r}\right\}$ of divergence-free vector fields.

Denote by $\mathrm{CL}(W)$ the cup-length of $W$, i.e., the maximal number of elements in $\mathrm{H}_{*>0}(W ; \mathbb{F})$ such that their cup-product is not equal to zero, maximized over all fields $\mathbb{F}$. Likewise, let $\mathrm{SB}(W)$ (the sum of Betti numbers) stand for $\sum_{j} \operatorname{dim}_{\mathbb{F}} \mathrm{H}_{j}(W ; \mathbb{F})$, maximized again over all $\mathbb{F}$.

In the spirit of the Arnold conjecture, we have

Theorem 2.2. Let $M$ and $W$ be as above, and furthermore assume that $\phi_{v}$ is regular. Then for any Hamiltonian $H$, the action functional $\mathcal{A}_{H}$ has at least $\mathrm{CL}(W)+1$ critical points. If $H$ is non-degenerate, the number of critical points is bounded from below by $\mathrm{SB}(W)$.

This theorem generalizes several previous results. Namely, when $r=1$ and $M=S^{1}$, this is the original Arnold conjecture as proved in [CZ]. When the target space $W$ is hyperkähler and the domain is either $M=\mathrm{SU}(2)$ or $M=\mathbb{T}^{3}$, the nondegenerate case of this theorem was originally proved in [HNS] using a version of Floer theory. When $M=\mathrm{SU}(2)$ and $W$ is hyperkähler or when $M$ is an arbitrary torus and $W$ is as above, both the degenerate and the non-degenerate case were established in $[\mathrm{GH}]$ by means of a finite-dimensional reduction method originating from [CZ] and similar, up to some technical details, to the one used here.

Remark 2.3. The proof of the theorem does not, in fact, make use of the requirement that $\mathcal{S}$ is a Clifford pencil (beyond some notational aspects), and thus the theorem holds for arbitrary symplectic pencils. However, in all cases where $\phi$ is regular known to the authors the pencil is Clifford; see Section 3.1.

Remark 2.4. As in [GH], Theorem 2.2 extends to non-compact quotients $W$ of $V$ without any significant changes in the proof. However, in the non-compact case, certain restrictions must be imposed on the behavior of the Hamiltonian $H$ at infinity and the lower bounds on the number of critical points may possibly depend on $H$. For instance, let us assume that a finite covering $W^{\prime}$ of $W$ is a Riemannian product of a flat torus and a Euclidean space $V^{\prime}$, e.g., $W$ is an iterated cotangent bundle of a flat manifold. Then it suffices to require the lift of $H$ to $M \times W^{\prime}$ to coincide, outside a compact set, with a non-degenerate quadratic form on $V^{\prime}$ with constant coefficients. In this case, the lower bounds on the number of critical points are again $\mathrm{CL}(W)+1$ and, respectively, $\mathrm{SB}(W)$.

\section{Regularity and the Clifford Condition}

3.1. Examples of Regular Frames. The analogy between the standard Dirac operator (see $[\mathrm{Hi}, \mathrm{LM}]$ ) and the operator $\phi_{v}$ suggests that $\phi_{v}$ need not be regular in general, unless the frame $v$ meets additional requirements. For $r=3$, the regularity of $\partial_{v}$ is studied in [Sa]. In this section, without attempting to analyze the regularity question in detail or depth, we briefly discuss some further examples where regularity or lack thereof can be established as a consequence of some other results.

Example 3.1 (Torus). Let $M$ be the torus $\mathbb{T}^{r}$ equipped with the standard volume form and let the frame $v$ be formed by vector fields with constant coefficients. (It suffices to assume that the vector fields commute: $\left[v_{i}, v_{j}\right]=0$ for all $i$ and $j$.) Then $\phi_{v}$ is regular for any Clifford pencil. 
Indeed, clearly, ker $\phi$ contains the constant functions. To show that all functions in the kernel are constant, we consider the operator

$$
\not^{2}=-\Delta+2 \sum_{i<j} J_{i} J_{j} L_{\left[v_{i}, v_{j}\right]}=-\Delta,
$$

where $\Delta:=\sum L_{v_{l}}^{2}$. This operator, in contrast with $\not$, is scalar - applying $\not^{2}$ to a function $f$ amounts to applying it to the components $u$ of $f$ with respect to some basis in $V$. Furthermore, $\not^{2}=-\Delta$ is (second order) elliptic and self-adjoint and the maximum principle holds for scalar solutions $u: M \rightarrow \mathbb{R}$ of the equation $\not^{2} u=0$, see [Ev, Section 6.4]. Since the domain $M$ of $u$ is a compact manifold without boundary, $u$ must be constant. To summarize, once $\not^{2} f=0$, every component of $f$ is a constant function, and hence $f$ is constant. Since, clearly, ker $\phi^{2} \supset$ ker $\phi$ (in fact, the two kernels coincide), we conclude that ker $\not$ contains only constant functions.

Example $3.2(\mathrm{SU}(2)$, following [Sa]). Let us identify $\mathrm{SU}(2)$ with the unit sphere in the space of quaternions $\mathbb{H}$ and let $v$ be the left-invariant frame such that $v_{1}=\mathbf{i}$, $v_{2}=\mathbf{j}$, and $v_{3}=\mathbf{k}$ at the identity. Set $V=\mathbb{H}$ with $J_{1}=\mathbf{i}, J_{2}=\mathbf{j}$ and $J_{3}=\mathbf{k}$. Then $\phi_{v}$ is regular; see [Sa] and also [GH, HNS].

On the other hand, if we take $v_{1}=2 \mathbf{i}, v_{2}=-\mathbf{j}$ and $v_{3}=-\mathbf{k}$ at the identity, keeping the rest of the data the same as above, the operator $\phi_{v}$ fails to be regular. Indeed, as is observed in [Sa], the natural inclusion $f: \mathrm{SU}(2) \rightarrow \mathbb{H}$ satisfies the equation $\phi_{v} f=0$.

Example 3.3 (Compact Lie Groups). Let $G$ be a compact $r$-dimensional Lie group with Lie algebra $\mathfrak{g}$. Fix a bi-invariant metric on $G$ and assume that the adjoint action lifts to a homomorphism $G \rightarrow \operatorname{Spin}(\mathfrak{g})$. Among the Lie groups with this property are tori, $\mathrm{SU}(n), \mathrm{SO}(2 n)$, the quaternionic unitary groups $\mathrm{Sp}(n)$, and of course all simply connected compact groups; see, e.g., [Sl]. Let $v$ be a left-invariant frame, which is orthonormal with respect to the bi-invariant metric. (For instance, the frames from Example 3.1 and the first frame considered in Example 3.2 meet this requirement, but the second frame in Example 3.2 does not.) Then $\phi_{v}$ is regular for any Clifford symplectic pencil.

To see this, first note that under our conditions on $v$, the operator $\phi_{v}$ can be identified with the standard Dirac operator (see, e.g., [LM] for the definition) with respect to the connection $\nabla$ on $G$ which is compatible with the bi-invariant metric and for which the frame $v$ is parallel, i.e., $\nabla_{\xi} v_{i}=0$ for all $i=1, \ldots, r$ and all tangent vectors $\xi$; see [Sl]. (This is not the Levi-Civita connection: $\nabla$ is not torsion-free unless $G$ is abelian. In fact, $T(\xi, \eta)=-[\xi, \eta]$, [S1], and clearly $\nabla$ is flat.)

By "additivity", it suffices to prove the result in the case where $V$ is an irreducible Clifford module. Furthermore, complexifying $V$ and extending $J_{i}$ 's to $V \otimes \mathbb{C}$ in a complex-linear fashion, we may assume that $V$ is a complex irreducible representation of $C l_{r}$. (When $r$ is even $V$ is unique and when $r$ is odd there are two such representations; [LM].) In this setting the regularity of the Dirac operator for $\nabla$ is established in [S1].

Example 3.4 (Generic Regularity). Let us fix $V$ and a Clifford pencil of rank $r$ on $V$. Assume that $M$ admits a regular frame $v$. Then the set of regular frames is open and dense in the $C^{\infty}$-topology in the collection of all divergence-free frames on $M$. 
The statement that regular frames form an open set is a consequence of the fact that surjectivity of a Fredholm operator is an open condition. To show that regular frames are dense, we argue similarly to the proof of [Sa, Lemma 1.3]. Let $w$ be an arbitrary frame, which we may assume to be non-regular. Then we claim that the frame $w+\epsilon v$ is regular for small $\epsilon>0$. Consider the kernel $K$ of the operator $\phi_{w}$ on the space of $W^{1,2}$-maps $M \rightarrow V$ with zero mean. Since $v$ is regular, the quadratic form $\left\langle f, \phi_{v} f\right\rangle$ is non-degenerate on $K$. Set $A(s)=\phi_{w+\epsilon v}$. The claim follows now from [Sa, Lemma A.2] asserting, roughly speaking, that an operator in a family of self-adjoint operators $A(s)$ is bijective whenever the quadratic form $\langle f, \dot{A}(0) f\rangle$ is non-degenerate on $\operatorname{ker} A(0)$ and $s>0$ is sufficiently small.

Remark 3.5. It is interesting to point out that the choice of a pencil plays no role in Examples 3.1-3.4. In fact, we do not have any example of a frame that would be regular for some pencils and non-regular for some others.

3.2. Clifford Condition Revisited. We conclude this discussion with a reformulation of the Clifford condition, which, we feel, better illuminates it from the symplectic-geometrical point of view. Namely, we claim that a symplectic pencil $\mathcal{S}$ is Clifford if and only if there exists an inner product $\langle$,$\rangle on V$ which is compatible (up to a factor) with all non-zero forms $\omega \in \mathcal{S}$. To be more precise, given an inner product $\langle$,$\rangle and a two-form \omega$, denote by $A_{\omega}$ the skew-symmetric matrix uniquely determined by the condition that $\omega(X, Y)=\left\langle A_{\omega} X, Y\right\rangle$ for all $X$ and $Y$ in $V$. We say that $\langle$,$\rangle is compatible with a symplectic pencil \mathcal{S}$ if for every $\omega \in \mathcal{S}$ we have $A_{\omega}^{2}=-\lambda I$ for some $\lambda \geqslant 0$ depending on $\omega$. In other words, every non-zero form $\omega \in \mathcal{S}$ becomes compatible with $\langle$,$\rangle in the standard sense after, if necessary, a$ rescaling. Note that when $\omega \neq 0$ we automatically have $\lambda>0$ since in this case $\omega$ is non-degenerate.

Proposition 3.6. A symplectic pencil $\mathcal{S}$ is Clifford if and only if there exists an inner product $\langle$,$\rangle which is compatible with \mathcal{S}$.

Proof. In one direction the assertion is obvious: a Clifford pencil admits an inner product compatible with it. Proving the converse, assume that $\langle$,$\rangle is compatible$ with $\mathcal{S}$. For $\omega$ and $\eta$ in $\mathcal{S}$, set $(\omega, \eta):=-\operatorname{tr}\left(A_{\omega} A_{\eta}\right)$. Clearly, this is a bi-linear symmetric pairing on $\mathcal{S}$, and due to the compatibility condition, (, ) is positivedefinite: $(\omega, \omega)=\lambda>0$ when $\omega \neq 0$. We can rewrite this as

$$
A_{\omega}^{2}=-\|\omega\|^{2} I \text {, }
$$

where $\|\cdot\|$ stands for the norm on $\mathcal{S}$ with respect to $($,$) . In particular, A_{\omega}$ is a complex structure if and only if $\|\omega\|=1$,

To prove that $\mathcal{S}$ is a Clifford pencil, it suffices to find a basis $\omega_{1}, \ldots, \omega_{r}$ of unit symplectic forms such that the operators $A_{\omega_{i}}$ anti-commute. We claim that this is true for any orthonormal basis. To establish this, it suffices to show that $A_{\omega}$ and $A_{\eta}$ anti-commute whenever $\omega$ and $\eta$ are orthogonal to each other with respect $($,$) . Consider two such forms, which we can assume to have unit norm, and set$ $\sigma=a \omega+b \eta \in \mathcal{S}$, where $a$ and $b$ are non-zero scalars. Then, by mutual orthogonality of these forms,

$$
\|\sigma\|^{2}=a^{2}\|\omega\|^{2}+b^{2}\|\eta\|^{2}=a^{2}+b^{2}
$$

and thus, by (3.1),

$$
A_{\sigma}^{2}=-\left(a^{2}+b^{2}\right) I
$$


On the other hand, since $A_{\sigma}=a A_{\omega}+b A_{\eta}$, we have

$$
A_{\sigma}^{2}=-\left(a^{2}+b^{2}\right) I+a b\left(A_{\omega} A_{\eta}+A_{\eta} A_{\omega}\right) .
$$

It follows that $A_{\omega} A_{\eta}+A_{\eta} A_{\omega}=0$, i.e., $A_{\omega}$ and $A_{\eta}$ anti-commute.

\section{Proof of Theorem 2.2}

The argument follows closely the finite-dimensional reduction method of Conley and Zehnder, [CZ], and its version for multi-dimensional time $M=\mathrm{SU}(2)$ or $\mathbb{T}^{r}$ introduced in $[\mathrm{GH}]$. However, we do not work with an explicit expression for $\phi$ obtained via Fourier analysis on a compact Lie group (which $M$ is not) relying on the Peter-Weyl theorem. Instead, we expand $f$ in eigenvectors of $\phi$ using the ellipticity of $\phi$ to reduce the problem to finite-dimensional Morse theory for generating functions. Beyond this point, the argument is quite standard, and hence we omit here some straightforward, minor technical details of the proof.

Let us first assume that $W$ is the quotient of a vector space $V$ by a lattice and, as a consequence, $W$ is a torus. We will discuss the modifications needed to deal with the general case at the end of the proof. (This step is essentially identical to its counterpart in $[\mathrm{GH}]$.)

Since $f$ is null-homotopic, it can be lifted to a map $\tilde{f}: M \rightarrow V$, where only the mean value $f_{0}$ depends on the lift. In other words, here we view $\mathcal{E}$ as an infinitedimensional vector bundle over $W$ with projection map $f \mapsto f_{0}$. This vector bundle is trivial and its fiber $\mathcal{F}$ is canonically isomorphic to the space of smooth maps $M \rightarrow V$ with zero mean. We can regard $\mathcal{E}$ as a sub-bundle in $W \times L_{0}^{2}(M, V)$.

Proposition 4.1. The operator $\not$ is elliptic on the space of $V$-valued functions on $M$ and self-adjoint with respect to the $L^{2}$-inner product on this space.

Proof. The operator $\not$ is elliptic if and only if the symbol $\sigma(\not)=\sum \lambda_{l} J_{l}$ is invertible for all non-zero (co)vectors $\lambda=\left(\lambda_{1}, \ldots, \lambda_{r}\right)$. Essentially by definition, this is true for any metric $\langle$,$\rangle , since the forms \omega_{l}$ generate a symplectic pencil. (In the Clifford case, we have $\sigma(\not)^{2}=-\left(\sum \lambda_{l}^{2}\right) I$, which also establishes ellipticity.) That $\not$ is self-adjoint can be easily shown using Stokes' theorem and the fact that the timemanifold $M$ is closed.

The next step in the proof of the theorem is decomposing $\mathcal{E}$ into the eigenspaces of $\not$. The fact that the eigenvalues go to infinity or negative infinity is then sufficient for the finite-dimensional reduction to go through. Note that we can view $\phi$ either as a linear operator on $\mathcal{F}$ or as a fiberwise linear operator on $\mathcal{E}=W \times \mathcal{F}$, independent of the point of the base. By the regularity assumption, the latter operator is fiberwise non-degenerate, which will be crucial for the proof of the theorem. (This is not just a consequence of ellipticity.)

Recall that the spectrum of a self-adjoint elliptic operator is real and countable and the eigenvalues tend to infinity and/or negative infinity; see, e.g., [LM, Chapter III]. Furthermore, the eigenspaces are finite-dimensional, mutually orthogonal, and the eigenvectors form a complete orthogonal system.

Denote by $\mathcal{F}_{N}$ the subspace in $\mathcal{F}$ spanned by all eigenvectors of $\not$ for eigenvalues with absolute value not exceeding $N$ and let $\mathcal{F}_{N}^{\perp}$ be the $L^{2}$-orthogonal complement of $\mathcal{F}_{N}$ in $\mathcal{F}$. Thus, $\mathcal{F}_{N}^{\perp}$ is spanned by all eigenvectors whose eigenvalues have absolute value greater than $N$. We can view $\mathcal{E}_{N}:=W \times \mathcal{F}_{N}$ as a subbundle in $\mathcal{E}$. It will also be useful to regard $\mathcal{E}$ as a vector bundle over $\mathcal{E}_{N}$ with fiber $\mathcal{F}_{N}^{\perp}$. 
Denote by $\mathcal{P}_{N}$ the (fiberwise) $L^{2}$-orthogonal projection of $\mathcal{E}$ onto $\mathcal{E}_{N}$ and by $\mathcal{P}_{N}^{\perp}$ the projection of $\mathcal{E}=\mathcal{E}_{N} \times \mathcal{F}_{N}^{\perp}$ onto the second component $\mathcal{F}_{N}$.

Our goal is to show that equation (2.3) has at least the desired number of solutions. Let $f=g+h$ with $g \in \mathcal{E}_{N}$ and $h \in \mathcal{F}_{N}^{\perp}$. Clearly, $f$ satisfies (2.3) if and only if

$$
\not g=\mathcal{P}_{N} \nabla H(g+h)
$$

and

$$
\not h=\mathcal{P}_{N}^{\perp} \nabla H(g+h) .
$$

Let us focus on the second of these equations with $g$ fixed and both sides viewed as functions of $h$, cf. [CZ] and [GH]. Since $\not$ is regular and by the definition of $\mathcal{F}_{N}^{\perp}$, the restriction of the operator $\not \partial$ to $\mathcal{F}_{N}^{\perp}$ is invertible and has only eigenvalues whose absolute value is greater than $N$. Denote the inverse of this restriction by $\phi_{N}^{-1}$. It is clear that (4.2) is equivalent to the equation

$$
h=\phi_{N}^{-1} \mathcal{P}_{N}^{\perp} \nabla H(g+h) .
$$

Note that the right hand side is now defined for all $h$ in the $L^{2}$-closure $\overline{\mathcal{F}}_{N} \frac{\perp}{}$ of $\mathcal{F}_{N}^{\perp}$, without any smoothness requirement. We claim that for sufficiently large $N$ and for any $g \in \mathcal{E}_{N}$, equation (4.3) (and hence (4.2)) has a unique solution $h=h(g)$ and this solution is smooth.

To show this, note first that, when $N$ is sufficiently large, $h \mapsto \phi_{N}^{-1} \mathcal{P}_{N}^{\perp} \nabla H(g+h)$ is a contraction operator on $\overline{\mathcal{F}}_{N}^{\perp}$ with respect to the $L^{2}$-norm. Indeed,

$\left\|\not_{N}^{-1} \mathcal{P}_{N}^{\perp} \nabla H\left(g+h_{1}\right)-\not \phi_{N}^{-1} \mathcal{P}_{N}^{\perp} \nabla H\left(g+h_{0}\right)\right\| \leqslant O(1 / N)\left\|\nabla H\left(g+h_{1}\right)-\nabla H\left(g+h_{0}\right)\right\|$.

(All norms are $L^{2}$ unless specified otherwise.) Furthermore, in obvious notation,

$$
\begin{aligned}
\left\|\nabla H\left(g+h_{1}\right)-\nabla H\left(g+h_{0}\right)\right\| & =\left\|\int_{0}^{1} \frac{d}{d s} \nabla H\left(g+s h_{1}+(1-s) h_{0}\right) d s\right\| \\
& \leqslant\left\|\nabla^{2} H\right\|_{L^{\infty}}\left\|h_{1}-h_{0}\right\| .
\end{aligned}
$$

Hence,

$$
\left\|\phi_{N}^{-1} \mathcal{P}_{N}^{\perp} \nabla H\left(g+h_{1}\right)-\phi_{N}^{-1} \mathcal{P}_{N}^{\perp} \nabla H\left(g+h_{0}\right)\right\| \leqslant O(1 / N)\left\|h_{1}-h_{0}\right\|,
$$

which shows that we can indeed choose $N$ sufficiently large such that the map $h \mapsto \phi_{N}^{-1} \mathcal{P}_{N}^{\perp} \nabla H(g+h)$ is a contraction.

The fact that the fixed point $h=h(g)$ of this operator is a smooth function is established by the standard bootstrapping argument and elliptic regularity. Namely, recall that, since $\phi$ is a first order elliptic operator (see Proposition 4.1), a solution $h$ of the equation $\phi h=y$ is of Sobolev class $H^{s+1}$ whenever $h$ and $y$ are $H^{s}$; see, e.g., [LM, p. 193]. We have $\not h=\mathcal{P}_{N}^{\perp} \nabla H(g+h) \in L^{2}=H^{0}$, and therefore $h \in H^{1}$. Now, since $H$ and $g$ are smooth, we also have $\mathcal{P}_{N}^{\perp} \nabla H(g+h) \in H^{1}$, and hence $h \in H^{2}$, etc.

Note also that as an immediate consequence of (4.3) and of the fact that $\nabla H$ is bounded, since $H$ is a function on a compact manifold, we have

$$
\|h(g)\|=O(1 / N) \text { and }\|\not h(g)\|=O(1) \text { uniformly in } g .
$$

These estimates will be used in the proof of Lemma 4.2 below.

From a more geometric perspective, $h(g)$ is the unique critical point of the action functional $\mathcal{A}_{H}$ on the fiber over $g$ of the vector bundle $\mathcal{E} \rightarrow \mathcal{E}_{N}$. Set $\Phi(g):=$ $\mathcal{A}_{H}(g+h(g))$. In other words, $\Phi$ is obtained from $\mathcal{A}_{H}$ by restricting the action functional to the section $g \mapsto h(g)$ of this vector bundle, formed by the fiber-wise 
critical points. Therefore, $g$ is a critical point of $\Phi$ if and only if $f=g+h(g)$ is a critical point of $\mathcal{A}_{H}$, i.e., a solution of (2.3), and every critical point of $\mathcal{A}_{H}$ is captured in this way. It remains to show that the generating function $\Phi$ on $\mathcal{E}_{N}$ has the required number of critical points.

The key feature of this function is that it is asymptotically (i.e., at infinity in the fibers of $\mathcal{E}_{N}$ ) a non-degenerate quadratic form. To be more precise, set

$$
\Phi_{0}(g)=\mathcal{A}(g)=\langle\not g, g\rangle_{L^{2}} \text { and } R=\Phi-\Phi_{0} .
$$

By definition, $\nabla \Phi_{0}(g)=\not g$. Hence, as has been pointed out above, the unperturbed action $\Phi_{0}$ is a fiberwise non-degenerate quadratic form since $\not$ is regular. (Here and throughout the rest of the proof, the metric on $\mathcal{E}_{N}=W \times \mathcal{F}_{N}$ is the product of the fiberwise $L^{2}$-metric and the metric on $W$.) Furthermore, the perturbation $R$ is small compared to $\Phi_{0}$ when $N$ is sufficiently large. To be more precise, we have

Lemma 4.2. Outside a compact set in $\mathcal{E}_{N}$, we have

$$
|R|+\|\nabla R\|<\left\|\nabla \Phi_{0}\right\|
$$

Remark 4.3. Lemma 4.2, combined with the non-degeneracy of $\Phi_{0}$, implies that the critical set of $\Phi$ is a compact subset of $\mathcal{E}_{N}$. As a consequence, the set of solutions of (2.3) (the critical points of $\mathcal{A}_{H}$ ) is also compact. Proving this fact directly, in the context of the hyperkähler Floer theory, is a rather subtle problem; cf. [HNS]. This is the reason we have chosen to provide a detailed proof of (4.5) below.

Proof of Lemma 4.2. To establish (4.5), note first that $H$ and $\nabla H$ are bounded, for $H$ is a function on a compact manifold. Therefore, the integral of $H$ makes a bounded contribution to $R$ and $\nabla R$, while the right hand side of (4.5) grows linearly as $g \rightarrow \infty$ in the fiber due to the non-degeneracy of $\Phi_{0}$. Thus, we can ignore $H$ in (4.5) and only need to estimate the growth of the difference

$$
R_{0}:=\mathcal{A}(g+h(g))-\mathcal{A}(g)=2\langle\not g g, h(g)\rangle+\langle\not h h(g), h(g)\rangle,
$$

or, to be more precise, of $\left|R_{0}\right|$ together with $\left\|\nabla R_{0}\right\|$. (It is worth pointing out that this is not equivalent to proving the lemma in the case where $H=0$. Even when the contributions of $H$ and $\nabla H$ to $R$ and $\nabla R$ are ignored, $H$ still enters the problem via the map $h$ which depends on $H$.)

To estimate the growth of $\left|R_{0}\right|$ and $\left\|\nabla R_{0}\right\|$, first observe that

$$
\left|R_{0}(g)\right| \leqslant O(1 / N)\left(\left\|\nabla \Phi_{0}(g)\right\|+1\right) .
$$

(Recall our convention from above that all norms, here and below, are $L^{2}$ unless specified otherwise.) This is an immediate consequence of (4.4).

In a similar vein, it is not hard to prove that

$$
\left\|\nabla R_{0}(g)\right\| \leqslant O(1)+O(1 / N)\left\|\nabla \Phi_{0}(g)\right\|
$$

by observing that the derivative of the function $g \mapsto h(g)$ is uniformly bounded by a constant $O(1 / N)$, as can be shown by differentiating (4.3) with respect to $g$. (See the calculation below.) Combining the upper bounds (4.7) and (4.8) with the fact that $\left\|\nabla \Phi_{0}(g)\right\|$ grows linearly with $g$ since $\Phi_{0}$ is non-degenerate, we see that (4.5) holds outside a compact set.

We conclude the argument by giving a detailed proof of (4.8). Differentiating (4.6), we have

$$
\begin{aligned}
d R_{0}(g)(w) & =2\langle\not \phi w, h(g)\rangle+2\langle\not g, D h(g) w\rangle \\
& +\langle\not(D h(g) w), h(g)\rangle+\langle\not h h(g), D h(g) w\rangle,
\end{aligned}
$$


where $w \in T_{g} \mathcal{E}_{N}$. To prove (4.8), we will bound every term on the right hand side of (4.9).

The first term: Using (4.4) and the fact that $\phi$ is self-adjoint, we have

$$
|\langle\not w, h(g)\rangle|=|\langle w, \not h(g)\rangle| \leqslant O(1)\|w\| .
$$

To deal with the remaining terms, we need to obtain an upper bound on the norm of the operator $D h(g): T_{g} \mathcal{E}_{N} \rightarrow T_{h(g)} \mathcal{E}$. By (4.3), we have, in obvious notation,

$$
\begin{aligned}
D h(g) w & =\left.\frac{d}{d s} \phi_{N}^{-1} \mathcal{P}_{N}^{\perp} \nabla H(g+s w+h(g+s w))\right|_{s=0} \\
& =\phi_{N}^{-1} \mathcal{P}_{N}^{\perp} \nabla^{2} H(g+h(g)) w+\not_{N}^{-1} \mathcal{P}_{N}^{\perp} \nabla^{2} H(g+h(g)) D h(g) w .
\end{aligned}
$$

Hence,

$$
\left[I-\phi_{N}^{-1} \mathcal{P}_{N}^{\perp} \nabla^{2} H(g+h(g))\right] D h(g) w=\phi_{N}^{-1} \mathcal{P}_{N}^{\perp} \nabla^{2} H(g+h(g)) w,
$$

and thus

$$
D h(g) w=\left[I-\not_{N}^{-1} \mathcal{P}_{N}^{\perp} \nabla^{2} H(g+h(g))\right]^{-1} \phi_{N}^{-1} \mathcal{P}_{N}^{\perp} \nabla^{2} H(g+h(g)) w .
$$

Note that as in (4.4), since $H$ is bounded together with all its derivatives, we have

$$
\left\|\phi_{N}^{-1} \mathcal{P}_{N}^{\perp} \nabla^{2} H(g+h(g))\right\|=O(1 / N)
$$

uniformly in $g$. Therefore,

$$
\|D h(g) w\|=O(1 / N)\|w\| .
$$

Now we are in a position to bound the remaining terms.

The second term: Recall that $\nabla \Phi_{0}(g)=\not g$. Using (4.11), we have

$$
|\langle\not g, D h(g) w\rangle| \leqslant O(1 / N)\left\|\nabla \Phi_{0}(g)\right\| \cdot\|w\| .
$$

The third and the fourth terms: These two terms are equal; for $\not$ is self-adjoint. Thus, by (4.4) and (4.11), we have

$$
\mid\langle\not(D h(g) w, h(g)\rangle|=|\langle\not h(g), D h(g) w\rangle| \leqslant O(1 / N)\|w\| .
$$

Combining the estimates (4.10), (4.12) and (4.13) for the individual terms with (4.9), we obtain the desired estimate (4.8). This concludes the proof of the lemma.

An argument similar to the proof of Lemma 4.2 shows that a critical point $g$ of $\Phi$ is non-degenerate if and only if $f=g+h(g)$ is a non-degenerate critical point of $\mathcal{A}_{H}$.

Finally, recall that whenever $\Phi=\Phi_{0}+R$ is a function on the total space of a vector bundle over an arbitrary closed manifold $W$ such that $\Phi_{0}$ is a fiberwise nondegenerate quadratic form and (4.5) holds, the function $\Phi$ has at least $\mathrm{CL}(W)+1$ critical points. Moreover, when $\Phi$ is Morse, the number of critical points is bounded from below by $\mathrm{SB}(W)$. This is a standard fact and we refer the reader to [CZ] for the original proof and to, e.g., [We2] for a different argument.

The general case, where $W$ is the quotient of $V$ by a group $\Gamma$, is treated exactly as in $[\mathrm{GH}]$. Namely, first recall that $\Gamma$ contains a finite-index subgroup $\Gamma^{\prime}$ consisting only of parallel transports, [Wo, p. 110]. Thus $W^{\prime}=V / \Gamma^{\prime}$ is a torus and the projection $W^{\prime} \rightarrow W$ is a covering map with a finite group of deck transformations, $\Pi=\Gamma / \Gamma^{\prime}$. The previous argument applies to the natural lift of the problem to $W^{\prime}$ and the entire construction is $\Pi$-equivariant. As a result, we obtain a vector bundle $\mathcal{E}_{N}^{\prime} \rightarrow W^{\prime}$ equipped with a $\Pi$-action covering the $\Pi$-action on $W^{\prime}$ and a $\Pi$-invariant 
function $\Phi^{\prime}$ on $\mathcal{E}_{N}^{\prime}$, which is asymptotically quadratic at infinity, i.e., $\Phi^{\prime}$ satisfies (4.5). The critical points of $\mathcal{A}_{H}$ for the original problem correspond to the $\Pi$-orbits of the critical points of $\Phi^{\prime}$. The quotient $\mathcal{E}_{N}=\mathcal{E}_{N}^{\prime} / \Pi$ is a vector vector bundle over $W$ and the function $\Phi^{\prime}$ descends to a function $\Phi$ on $\mathcal{E}_{N}$. (The quotient $\mathcal{E}_{N}$ and the function $\Phi$ are smooth, for the $\Pi$-action on $\mathcal{E}_{N}^{\prime}$ is free as an action covering a free action on $W^{\prime}$.) The critical points of $\Phi$ are in one-to-one correspondence with the critical points of $\mathcal{A}_{H}$ for the original problem and $\Phi$ is also asymptotically quadratic at infinity. The theorem now follows as before from the lower bounds on the number of critical points of $\Phi$.

\section{REFERENCES}

[CZ] C. Conley, E. Zehnder, The Birkhoff-Lewis fixed point theorem and a conjecture of V.I. Arnold, Invent. Math., 73 (1983), 33-49.

[Ev] L.C. Evans, Partial Differential Equations, Graduate Studies in Mathematics, vol. 19, Second edition, AMS 2010.

[FZ] L. Florit, W. Ziller, Topological obstructions to fatness, Preprint 2010, arXiv:1001.0967.

[GH] V. Ginzburg, D. Hein, Hyperkähler Arnold Conjecture and its Generalizations, Preprint 2011, arXiv:1105.0874; to appear in Internat. J. Math.

[GHJ] M. Gross, D. Huybrechts, D. Joyce, Calabi-Yau Manifolds and Related Geometries, Lectures at a Summer School in Nordfjordeid, Norway, June 2001, Springer-Verlag, Berlin, Heidelberg, 2003.

[Hi] N. Hitchin, Harmonic spinors, Advances in Math., 14 (1974), 1-55.

[HNS] S. Hohloch, G. Noetzel, D. Salamon, Hypercontact structures and Floer theory, Geom. Topol., 13 (2009), 2543-2617.

[HP] J.-S. Huang, P. Pandžić, Dirac Operators in Representation Theory, Birkhäuser Boston, Inc., Boston, MA, 2006.

$[\mathrm{Hu}] \quad$ D. Husemoller, Fibre Bundles, Springer-Verlag, New York, 1994.

[Jo] D. Joyce, Manifolds with many complex structures, Quart. J. Math. Oxford Ser. (2), 46 (1995), 169-184.

[LM] H.B. Lawson, M.-L. Michelsohn, Spin Geometry, Princeton Mathematical Series, 38, Princeton University Press, Princeton, NJ, 1989.

[Mo] R. Montgomery, A Tour of Subriemannian Geometries, their Geodesics and Applications, Mathematical Surveys and Monographs, vol. 91, American Mathematical Society, Providence, RI, 2002.

[Sa] D. Salamon, The three dimensional Fueter equation and divergence free frames, Preprint 2012, arXiv:1202.4165.

[Sl] S. Slebarski, Dirac operators on a compact Lie group, Bull. London Math. Soc., 17 (1985), 579-583.

[MS] A. Moroianu, U. Semmelmann, Clifford structures on Riemannian manifolds, Preprint 2009, arXiv:0912.4207.

[We1] A. Weinstein, Fat bundles and symplectic manifolds, Adv. in Math., 37 (1980), 239250 .

[We2] A. Weinstein, $C^{0}$ perturbation theorems for symplectic fixed points and Lagrangian intersections, in South Rhone seminar on geometry, III (Lyon, 1983), pp. 140-144, Travaux en Cours, Hermann, Paris, 1984.

[Wo] J. Wolf, Spaces of Constant Curvature, Fifth edition, Publish or Perish, Inc., Houston, TX, 1984.

Department of Mathematics, UC Santa Cruz, Santa Cruz, CA 95064, USA

E-mail address: ginzburg@ucsc.edu

E-mail address: dhein@ucsc.edu 